\title{
The clinicopathological profile of malignancies in the ampullary region
}

L. B. D. J. Siriwikum, N. K. Samarakoon, S. K. Liyanage, B. A. G. G. Mahendra, R. M. U. S. Rathnayake and S. J. de S Hewavisenthi

Department of Pathology, Faculty of Medicine, University of Kelaniya, Sri Lanka

DOI: http://doi.org/10.4038/jdp.v11i2.7717

\section{Introduction}

The ampulla is a poorly defined region, both anatomically and histologically, affected by malignancies arising in the duodenum, distal common bile duct, head of the pancreas and those centered around the ampulla. These tumours are collectively considered as ampullary carcinomas. The objective of this study was to describe the clinicopathological features of malignancies arising in the ampullary region.

\section{Methodology}

This was a retrospective study of 42 histopathology reports of resection specimens of malignant tumours in the ampullary region, received at the Department of Pathology, Faculty of Medicine, Ragama, over a period of four years. Clinical data and pathological features were collected and analyzed.

\section{Results}

The male to female ratio was $8: 13$. The median age of the patients was 57 years (range 15 -77). The single most common presentation was jaundice 15/42 (35.71\%), but a majority of patients $(16 / 42,38.09 \%)$ presented with multiple symptoms (abdominal pain, weight loss and jaundice). The average duration of symptoms was 2.8 months. Malignancies included 5 biliary (B), 8 duodenal (D), 11 pancreatic (P) and 18 ampullary carcinomas (A). The histological types included adenocarcinoma 34/42 (80.95\%), neuroendocrine tumours $5 / 42$ (11.9\%) and other types 3/42(7.14\%) which included solid pseudopapillary neoplasm, acinar cell carcinoma of the pancreas and gastrointestinal stromal tumour. A majority of adenocarcinomas were moderately differentiated, i.e., 27/34 (B-4/5, D-4/5, P-5/8, $A-14 / 16)$. Lymphovascular invasion was seen in $11 / 34$ (B-1/5, D-2/5, P-3/8, A-5/16) and perineural invasion was identified in 12/34 (B$2 / 5, D-2 / 5, \mathrm{P}-5 / 8, \mathrm{~A}-3 / 16)$. The most common stage at presentation was IIB (T1/T2/T3, N1, MO - TNM classification - UICC).

\section{Conclusion}

Ampullary malignancies were more common among females. The median age was 57 years. The majority was moderately differentiated adenocarcinomas with lymphovascular and perineural invasion and the commonest stage at presentation was IIB. 